\title{
Disentangling the Effects of Reproductive Behaviours and Fertility Preferences on Child Growth in India
}

\author{
Md Juel Rana ${ }^{1}$, John Cleland ${ }^{2}$, T.V. Sekher ${ }^{3} \&$ Sabu S. Padmadas ${ }^{4}$
}

${ }^{1}$ Centre for the Study of Regional Development, School of Social Sciences, Jawaharlal Nehru University New Delhi, India. Email ID: juel14_ssf@jnu.ac.in / jranajnu@gmail.com $\underline{\text { and }}$

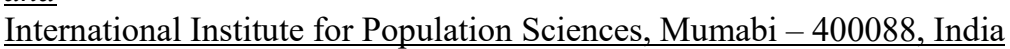

${ }^{2}$ Population Studies Group, London School of Hygiene and Tropical Medicine, University of London, United Kingdom. Email ID: john.cleland@1shtm.ac.uk

${ }^{3}$ Department of Population Policies and Programme, International Institute for Population Sciences Mumbai, India. Email ID: sekher@iips.net

${ }^{4}$ Department of Social Statistics and Demography, Faculty of Social Sciences, University of Southampton Southampton, UK. Email ID: S.Padmadas@soton.ac.uk 


\begin{abstract}
We analysed population data from the 2015-16 National Family Health Survey to disentangle the underlying intricate effects of reproductive behaviours and fertility preferences on child growth. We hypothesised that birth interval would have a stronger association with stunting than sibsize and these effects would be moderated by whether the child was wanted or unintended (mistimed or unwanted). Regression analyses showed strong and equal effects of short birth interval and sibsize on stunting, when adjusted for potential confounders and unobserved heterogeneity between-mothers. There were no statistical associations between stunting and mistiming/unwantedness of index children, suggesting the absence of discrimination against such children. We conclude that while fertility preferences have no effect, reproductive behaviours exert significant influence in predicting child growth. Sibsize have been falling for many years in India but inter-birth intervals have remained largely unchanged. The results underscore the need for strengthening uptake of reversible contraceptives for improved birth interval.
\end{abstract}

\title{
Keywords
}

Child growth; Stunting; Unintended; Mistimed; Unwanted; Preceding Birth Interval; Sibsize; Fertility preference; Reproductive behaviour; India 


\section{Introduction}

This paper contributes to new knowledge in several ways. First, unlike previous studies, we were able to confirm results by taking into account unobserved mother-level heterogeneity. Second, where most studies used parity or birth order as a predictor, we considered the number of surviving siblings (sibsize) and we were able to compare the effects of family size on stunting via the economic pathway of diluted resources per child, with the effects of preceding interval via the biological pathway of maternal depletion. Third, our analyses overcome the well-established bias, where mothers classify children as unwanted at time of conception, by using an alternative measure based on total desired family size. Finally, our study is one of very few to investigate whether or not reproductive attitudes mediate the effects of preceding interval or family size on growth of children.

Despite substantial improvements in under-five mortality, India continues to have the largest share of undernourished children in the world (UNICEF 2017). Two in five children in India are stunted (IIPS and ICF 2017). Undernourished children have elevated risks of infections, cognitive and neurological impairment, metabolic disorders, chronic disease and low economic productivity in later life (Gutbrod et al. 2000; Schaible and Kaufmann 2007; Victora et al. 2008; Hoddinott et al. 2013). The need for a better understanding of the circumstances that lead to poor growth in Indian children is a national health priority.

The first aim of this paper is to assess the linkages between reproductive behaviour and the risk of stunting in under-five children in India. Specifically, we examine two indicators of behaviour: preceeding birth interval length and sibsize, that is the number of surviving children in the family. These two components of family building, of course, are related. Women with large numbers of children are likey to have experienced shorter inter-birth intervals than those with small family but the association is not so close as to prevent independent examination of both. An important unresolved issue for India is the relative strength of these two risk factors for stunting. The second aim of this paper is to assess links between unintendedness and stunting, and to establish whether or not reproductive attitudes mediate, or attenuate, associations between birth interval and sibsize and stunting.

A substantial literature, mostly based on the analysis of Demographic and Health Surveys (DHSs) show evidence on the associations between child growth and birth interval and sibsize, or its closely related but imperfect surrogate, birth order. While the positive effect of 
preceeding birth interval length on child survival is firmly established, evidence of its effect on stunting is less clear. Using the pooled DHS data from 52 different countries between 2006 and 2012, Rutstein, and Winter (2014) found a negative association between preceding birth interval length and stunting, after adjusting for a wide range of possible confounders. Compared with preceding birth to conception intervals of 36-47 months, the adjusted odds of stunting rose monotonically with shorter intervals, with odds of 1.39 for intervals of less than six months. The causal pathways are probably biological, including poor foetal growth and prematurity, owing to maternal depletion, though sibling competition and cross-infection may also contribute (Boerma and Bicego 1992; Conde-Agudelo 2012). However, effects on interval length appear to be context-specific. An extensive literature review by Dewey and Cohen (2007) showed that, out of 50 studies conducted in developed and less developed countries, about a half found no significant association between preceding birth interval and child nutritional outcomes, while the rest found the expected adverse effect of short intervals. This review also found that the association between succeeding birth interval and child nutrition outcomes, both negative and positive.

The literature on the effects of sibsize and birth order, is similarly extensive. An analysis of height for age in children aged 3-36 months in 15 countries based on DHS data found moderate associations with the presence of a sibling aged under five years of age but very small associations for the presence of older siblings, suggesting that short intervals between births may be a more important influence on growth than the number of siblings (Desai 1995). However, after controlling for preceding interval length, the effect of an under-five sibling on stunting of the index child remained significant in 10 of the 15 surveys. Resource dilution is the obvious explanation for any effects of sibsize on child growth. The pooled analysis of Rutstein and Winter (2014) found the odds ratios of stunting by birth order to be trivial after adjustment for birth interval length and other factors; compared with birth orders 1-2, the odds of stunting for birth orders 3-4 was 1.03, rising to 1.08 for orders 5-6. This result suggests that birth interval may be a more important influence on stunting than the number of siblings, though, as discussed below, birth order is not synonymous with sibsize.

A limited number of Indian studies have also found mixed results of association between reproductive behaviours and child stunting. Preceding birth interval and birth order both had significant effects on child stunting in a study using National Family Health Survey (NFHS3) data (Rana and Goli 2018). This study found that children born at the intersectional axes of 
higher birth order $(3+)$ and short birth interval ( $<24$ months) had the highest risk of child stunting. Using the same dataset and applying the additive quintile regression model, Fenske et al. (2013) found that preceding birth interval and birth order had non-linear positive and negative relationship, respectively, with height-for-age of the children under-five. Another study from a city in Madhya Pradesh showed that the risk of stunting was about twice among the children of birth interval $<24$ months than those with $48+$ months (Shahjada et al. 2014). Analysis of data from an urban slum of a city in Uttar Pradesh found that the likelihood of stunting was thrice among the children with third or higher birth order than second or lower birth order (Srivastava et al. 2012).

A recent study by Jayachandran and Pande (2017) found that children with higher order birth are more likely to be stunted and the gradient of stunting by birth order was steeper in India than in Sub-Saharan Africa. They argued that later born children suffer discrimination in terms of resource allocation due to favouritism or preference towards the elder sons. In a reanalysis of the same data, Spears et al. (2019) showed that the omission of sibsize in the anaysis was crucial. Indeed, when sibsize was included they found that higher order births were less, not more, likely to be stunted. In this paper we use sibsize as a preferable indicator of possible resource contraints within households rather than birth order.

A major problem with any assessment of the association between birth intervals or sibsize and child welfare is endogeneity (Öberg 2017). Couples who choose to have large families may differ in unmeasured attitudes and behaviour from couples who choose to have small families and these unmeasured factors may influence child welfare, including linear growth. We address this problem in two ways. The first is to apply a mother fixed effect model, which is possible in the case of birth interval. The second is to introduce into the analysis fertility preferences, in recognition that reproductive choice is far from perfect in India. It has been estimated that about half of all pregnancies in India are unintended, because of lack of access, broadly defined, to contraception (Singh et al 2018). Though over half are terminated, a substantial minority of unintended pregnancies are carried to term. The introduction of this perspective allows us, for instance, to compare the link between sibsize and stunting for children who were wanted (and thus a consequence of conscious choice) and those who can be classified as unwanted (as a consequence of lack of contraceptive access). To the extent that the gradient in stunting is similar for both wanted and unwanted children, confidence in a causal link will be increased. 
Of course, unintendedness or unwantedness of births may have its independent effect on child welfare, including growth, because of conscious or unconscious discrimination. The results of multi-country analyses are inconclusive on this (Montgomery and Lloyd 1997; Marston and Cleland 2002). Evidence from Bangladesh is also mixed. A study using the DHS data found a linkage between unwantedness and childhood stunting, while another study using the longitudinal data of the Matlab surveillance site observed no effect of unwantedness on child survival (Rahman 2015; Bishai et al. 2015). No effect of intendedness on stunting was found in Malawi but a study in Ethiopia showed that unintended children are more likely to be stunted than their counterparts (Baschieri et al. 2017; Shaka et al. 2020). A review by Gipson et al. (2008) confirmed the paucity of evidence and the conflicting results emerging from the small number of studies.

In India, the evidence is also rather limited. Singh et al. (2012), using cross-sectional data from the second round of NFHS, found that births reported by mothers as unintended received less prenatal care, had low vaccination rates and experienced a higher risk of neonatal death than intended births. Evidence from a follow-up study also showed a positive association between pregnancy unintendedness and adverse child health outcomes in rural India (Singh et al. 2013). Another analysis using longitudinal data from the Young Lives Study reported that unintended births were likely to be associated with the poor childhood development (Singh et al. 2017). However, these findings are open to doubt because other potential confounding factors such as birth interval, family size and economic status of the household were not systematically taken into account.

The mixed nature of the available evidence may stem in part from problems in the measurement of intendedness of births. Most of the studies cited above used retrospective data. Mothers were asked whether each of their recent births was wanted, unwanted or mistimed at the time of conception. NFHS asked two questions for assessing respondents' fertility intentions: "When you got pregnant with (NAME), did you want to get pregnant at that time?’ If the respondents answered 'yes', then the birth is considered as 'wanted'. If the respondents answered 'no', then the survey asked a follow-up question: "Did you want to have a baby later on, or did you not want any (more) children?” If the response was 'later', then the birth was classified 'mistimed' and for those who responded 'no more children', then the birth was classified 'unwanted'. Their responses could be affected by post-factum rationalisation; mothers might be reluctant to classify young children as unwanted, and, perhaps to a lesser extent, mistimed. This assertion 
is based on evidence from two prospective studies in India and others elsewhere; many women who asserted that they wanted no more children at the baseline survey later reported the births as wanted (Roy et al. 2008; Speizer et al. 2013). Rationalisation is not the sole explanation. It is likely that the prospective measure (Do you want any more children?) and the retrospective measure are capturing different dimensions. The first is an abstract planning concept, which may be influenced by the views of the spouse and relatives, whereas the second is likely to be a more personal emotional response to an event that has already occurred.

An alternative way to identify the unwanted births is to make use of the question on total desired family size. The relevant question in NFHS-4 is "If you could go back to the time you did not have any children and could choose exactly the number of children to have in your whole life, how many would that be?" A comparison of the total number of desired family size (DFS) with actual number of surviving children (AFS) will allow us to identify children born in excess of the desired number who may then be classified as unwanted. For instance, a child with two older siblings born to a mother who stated a desired size of two is classified as unwanted (DFS $<$ AFS). This measure also has limitations. Women may adjust upwards their desires to enforce consistency with the actual number of children. Preference for sex of the child adds further complication, particularly in Indian context known for son preference. For instance, a woman may state a two-child family as ideal but, if the first two children are of the same sex, she may want a third.

Based on Indian and international evidence, we hypothesise that the association between birth interval and stunting will be stronger than the association with sibsize, and that fertility attitudes will moderate these associations.

\section{Data and Methods}

Data

The data for this study were drawn from the recent round of the National Family Health Survey (NFHS-4), Indian equivalent of DHS, conducted during 2015-16 in India. The NFHS-4 collected data on key health and family welfare indicators through face to face interviews with 699,686 ever-married women of reproductive age who were selected using two-stage systematic random sampling (IIPS and ICF 2017). In addition, the NFHS-4 collected anthropometric and other health related data on 259,627 children born to these women in the last five years. Of these, height and weight measurements were available only for 236,455 
children. The final sample included 203,313 children, after excluding twin births and children whose current age was less than 6 months at the time of the survey. For the analysis of preceding birth interval, 32 children born following an implausibly short interval of less than seven months, and 1,407 cases where the preceding birth interval was more than ten years were dropped, since their inclusion may produce skewed results (see Appendix Table A1).

\section{Outcome variable}

Our outcome variable of interest was stunting measured in terms of z-scores representing height for age of children under five years based on a WHO reference population (WHO Multicentre Growth Reference Study Group and de Onis 2006). Stunting is the best single indicator of child growth impairment, which stems from inadequate diet and/or repeated infections. We defined stunting as a binary variable, coded as 1 for those children who fall less than two standard deviations of the median of the reference population.

\section{Explanatory variables}

The primary variables of interest of reproductive behaviour were: length of the preceding birth interval and sibsize. The preceding birth interval of the children was coded into first-order births (no interval), $>48$ months, 37-48 months, $25-36$ months and $<25$ months. Sibsize was grouped into 1, 2, 3, 4 and 5+ living children born to the same mother, including those who are usually older but may also be younger than the child under investigation (the index child). Thus, if there are two under-five children in a household, their sibsize will be the same. We considered two indicators to measure fertility preferences: whether or not the child is classified by the mother to be the result of a mistimed or unwanted conception and whether or not the child is in excess of total desired size and can thus be classified as unwanted at the time of conception.

\section{Confounding variables}

The analyses adjusted for a set of potential confounders at the child, mother, household and regional levels. The child level variables included: current age of the child grouped in months (6-11, 12-23, 24-35, 36-47 and 48-59) and sex of the index child. The maternal variables included: age of mothers at the time of birth of index child grouped in years $(<20,20-24,25-$ 29, and 30+), maternal height $(<145 \mathrm{cms}$ and $145 \mathrm{cms}$ and above), mother's education (primary, secondary, and higher) and current working status. The household level variables included are place of residence (rural and urban), religion (Hindu, Muslim and Others), caste 
(Scheduled Castes [SC], Scheduled Tribes [ST], Other Backward Castes [OBC] and others) and wealth quintiles of the households (poorest, poor, middle, rich and richest).

\section{Statistical analyses}

We fitted a set of binary logistic regression models to estimate the unadjusted and adjusted effects of the preceding birth interval, sibsize, and reproductive attitudes on child stunting. The adjusted effects of preceding birth interval on stunting by birth intendedness and the effects of sibsize on stunting by the difference between actual and desired family size have been estimated. A stratified analysis on the effects of sibsize by whether the child exceeds desired family size or not is shown later in the results section (see Table 4). The bivariate and multivariate estimates were adjusted for national representative women sample weight. All the analyses were carried out using Stata v16.0 (StataCorp, College Station, Texas 77845 USA).

The methods described above do not take into account the unmeasured maternal characteristics that may affect both maternal reproductive health behaviours and child health outcomes (Molitoris 2018). To overcome the potential heterogeneity between mothers, a separate 'within family' analysis was performed which was restricted to families with two or more under-five children. A total of 30,997 children whose mothers have at least one stunted and one normal child were eligible for this sub-analysis. Out of the total 14,815 mothers fulfilling this condition, 13,462, 1,335 and 16 mothers have two, three and four children, respectively. Using this sample, a binary logistic regression and a conditional binary logistic regression models were applied to assess the association between preceding birth interval and childhood stunting. The conditional logistic regression model was not possible for sibsize because, by definition, children have the same size. A conditional binary logistic regression model (clogit) or motherlevel fixed effect model was applied to assess the influence of unmeasured heterogeneity on the association between preceding birth interval and stunting of children.

A model that has fixed (non-random) parameters is called a fixed-effects model. A clustered data (like our data i.e. mothers with more than one child) is suitable for the fixed effect model. The logistic regression model has individual intercepts that allows for unobserved motherconstant heterogeneity. As maximum likelihood estimation with a dummy variable for each individual can result in estimates that are inconsistent, a conditional maximum likelihood approach can be used which does not give estimates of the individual fixed effects but gives estimates of the regression parameters of interest, which for logistic regression when 
exponentiated can be interpreted as odds ratios from the standard logistic regression model (McFadden, 1984; Allison, 2009). Thus, this model allows to estimate the odds ratios of child stunting by preceding birth interval controlling for the unobserved mother-level heterogeneity.

\section{Results}

\section{Descriptive statistics}

Table 1 presents the summary statistics of key explanatory variables. Of 203,313 births, 37 percent were first born, 17 percent were born after an interval of two years or less, and 20 percent after an interval of two to three years. About 37 percent of families had three or more children at the time of interview. Only about five percent of births were reported by mothers as mistimed. This proportion increases from two percent for children born after long birth intervals to nine percent among those born within two years or less of an older sibling (Appendix Table A1). A similarly small proportion were reported as unwanted and this proportion increases from less than one percent for first born children to 24 percent for those with four or more older siblings. About 27 percent of children were born in excess of desired family size. Over half of third or higher birth order children were in excess of total desired family size. The descriptive statistics of all study variables are presented in Appendix Table A2.

Table 1 also presents the prevalence of childhood stunting by preceding birth interval, sibsize and the intendedness of births. The prevalence of stunting falls monotonically with increasing birth interval from 49 percent for those with an interval of two years or less to 36 percent for those with an interval of more than four years. Similarly, stunting decreases as sibsize decreases from 55 percent among five children families to 32 percent among singleton families. The percentage of stunting is almost the same for children reported by mothers as wanted or mistimed (39-41 percent) but increases to 48 percent for those who were unwanted. The prevalence of stunting is 38 percent for those whose mothers have a family size less than or equal to their desired family size and 46 percent for those whose actual family size is higher than the desired.

\section{Preceding birth interval and child growth}

Table 2 presents the unadjusted and adjusted odds of stunting from the binary logistic models by the preceding birth interval, succeeding birth and mothers' classification of birth intendedness. The unadjusted model shows significant associations between interval length, 
the occurrence of a succeeding birth and whether the index child was classified as unwanted at time of conception but no association with births classified as mistimed. The second model includes preceding birth interval, succeeding birth and intendedness. The effects of interval length attenuate slightly but not significantly, the effects of unwantedness attenuate significantly, while the effects of a succeeding birth increase. We investigated whether or not breastfeeding status might explain the link between a succeeding birth and stunting. Whereas almost all index children with a younger sibling have been weaned, over half of last-born children were still being fed at the breast. However, we found that stunting was less prevalent in last born children who had been weaned than in those still breastfeeding (results not shown). The fully adjusted results in the third model indicate significant attentuation of interval length effects. Compared with an interval length of more than four years, the risk of stunting remains higher among children with a preceding birth interval of 37-48 months (OR: 1.13, CI: 1.081.17), 25-36 months (OR: 1.27, CI: 1.22-1.31), and <25 months (OR: 1.45, CI: 1.40-1.50). In this third model, no link between stunting and wantedness or succeeding birth was found.

\section{Sibsize and child growth}

Table 3 shows the effects of sibsize and unwantedness, as measured by the difference between desired and actual family size, on child stunting. The unadjusted results show sharp increases in stunting as sibsize increases and a large difference between children born in excess of desired family size and others. After adjustment for desired versus actual size, the effects of sibsize actually increase slightly but the direction of effects of desirability of the child reverses. These effects attenuate after full adjustment in the third model though remain statistically significant. Compared with one child families, the odds of stunting are higher among mothers who have two (OR: 1.08, CI: 1.04-1.13), three (OR: 1.22, CI: 1.16-1.29), four (OR: 1.35, CI: 1.27-1.44), and five or more children (OR: 1.52, CI: 1.42-1.63). The strength of assocation between stunting and sibsize is similar to those observed for interval length. Unexpectedly, the odds of stunting in the fully adjusted model remain slighly lower for undesired than for desired children.

Table 4 presents the unadjusted and adjusted effects of sibsize on stunting stratified by the difference between DFS and AFS. The unadjusted gradient in stunting is closely similar. The results from adjusted models show that among the children of mothers who have higher AFS than DFS, the likelihood of stunting is higher among the children with sibsize two (OR: 1.24, CI: 1.07-1.42), three (OR: 1.45, CI: 1.26-1.68), four (OR: 1.58, CI: 1.36-1.83) and five or more 
(OR: 1.77, CI: 1.52-2.06) as compared to sibsize one. The increasing risk of childhood stunting from sibsize one to five or more is lower among the children whose mothers have AFS less than or equal to DFS, compared to the children whose mothers have higher AFS than DFS but the gradient with increasing sibsize is similar.

A separate analysis for the preceding birth interval is carried out within a restricted sample of those whose mothers have at least two children in the last five years with second and higherorder births (Table 5). The addition of mother-fixed effects made little difference to the log odds of stunting by interval length.

\section{Discussion}

Contrary to expectations, we found that the effects of birth interval and sibsize on child growth were similar in magnitude. The percent of children who were stunted rises monotonically from 36 percent among those with a preceding birth interval of more than four years to 49 percent among those with an interval of two years or less. Adjustment for a battery of potential confounders including sibsize, mother's height, education and household wealth made little difference to the log odds of stunting by interval length, suggesting that the adverse impact of a short interval is broadly similar across socio-economic strata. The mother fixed effect model strongly suggests that unmeasured differences between mothers with varying birth interval patterns cannot account for the link between spacing and child growth.

The causal pathways linking short intervals to perinatal, infant and child outcomes are not clearly established but may include maternal depletion, competition for maternal attention and household resources, and cross-infection (Boerma and Bicego 1992; Conde-Aguudelo et al. 2012). The fact that a higher risk of stunting persists among the children who have short birth interval after adjustment for sibsize suggests that maternal depletion, the lack of time for a mother to recover from lost nutrients during the previous pregnancy, delivery and breastfeeding, may be the main mechanism rather than cross-infection or competition. Previous research has shown that short intervals are associated with prematurity and low birth weight which are likely to result in stunting (Conde-Agudelo et al. 2005; DaVanzo et al. 2008).

Our findings have important policy implications. Unlike many low- and middle-income countries, birth interval in India has changed little over the years (Casterline and Odden 2016). Between 1993 and 2016, the percent of second and higher order births that occur within 24 
months of the previous birth has remained unchanged at around 27 percent, with nearly 60 percent occurring in less than 36 months (Appendix, Figure 1A). The reason for this stability is evident. Historically, the Indian family planning programme has emphasised sterilization over other methods and the recent attempts to promote long acting reversible methods have not yet had the desired impact (Srinivasan 2017). So what needs to be done? Promoting reversible contraceptives with more effective campaigns and follow up services with adequate quality of care will be required.

The unadjusted effects of sibsize on childhood stunting were large and consistent with the pervasive association between number of children and poverty. After adjustment for confounders, the effects of sibsize attenuated more than was the case for interval length but nevertheless remain as large as those for interval. These results contrast strongly with those from an analysis of 45 DHS countries by Rutstein and Winter (2014) for reasons that are unclear but may include a lower ability in India than in other countries to increase income in response to a growing family or to seek support from a wider family network. We addressed the problem of endogeneity by a stratified analysis, according to whether or not the index child was unwanted. The gradient in stunting with increased sibsize was very similar. This result enhances a causal interpretation. For the wanted group, it can be argued that couples are choosing to prioritise a large family over the welfare of individual children in terms of nutrition and health care but the same argument is much less plausible for the unwanted group. It is true that in India, as elsewhere, poor couples are more likely to have higher fertility, and thus larger families than rich couples. They are also more likely to unwanted children. NFHS-4 shows that the gap between actual and wanted fertility narrows from 0.9 births for the poorest quintile to 0.1 births in the richest. Though we adjusted for a wide range of covariates, it remains possible that the association between sibsize and stunting has been affected by residual confounding.

In our analysis, reproductive attitudes, as measured by mothers' response on unintendedness of the pregnancy or the comparison between reported ideal and actual family size, had no effect on stunting after adjusting for reproductive behaviour. This result is consistent with much of the international literature but contradicts previous findings from India (Singh et al. 2017). The difference may be explained by the larger number of control factors used in this analysis than by Singh and colleagues. It may also reflect the particularly weak measurement of retrospective pregnancy-intendedness in NFHS-4 (IIPS and ICF 2017). Only about five percent of births were declared by mothers to be the outcome of mistimed pregnancy and a similar proportion 
to be the result of an unwanted pregnancy, much lower than what was found in earlier rounds of NFHS. However, the measure of unwantedness based on desired family size, which may be less prone to underreporting, yielded a similar result. The high incidence of abortion in India is relevant in this context. It has been estimated that well over half of all unintended pregnancies in India are terminated (Singh et al. 2018). Thus, it is most likely that the pregnancies carried to term are either intended and welcome or only mildly unwelcome.

We conclude that both birth intervals and number of children in a family raise the risk of inadequate growth in children by a substantial and approximately equal degree. Contrary to expectations we found no evidence that unintended or unwanted children are particularly vulnerable to stunting. Thus the link between the number of children or short intervals is not attributable to parental attitudes towards particular children whose addition to the family may have been undesired.

\section{Acknowledgements}

This work was undertaken with support from the Population Council through its project entitled "Policy Research and Advocacy for Strategic Investment in Maternal, Newborn and Child Health in India" supported by the John D. and Catherine T. MacArthur Foundation (Grant ID: G-109245-0).

\section{References}

Adhikari, Ramesh P., Manisha Laxmi Shrestha, Ajay Acharya, and Nawaraj Upadhaya. 2019. Determinants of stunting among children aged 0-59 months in Nepal: findings from Nepal Demographic and health Survey, 2006, 2011, and 2016. BMC Nutrition 5(1):37.

Akram, Raisul, Marufa Sultana, Nausad Ali, Nurnabi Sheikh, and Abdur Razzaque Sarker. 2018. Prevalence and determinants of stunting among preschool children and its urbanrural disparities in Bangladesh. Food and nutrition bulletin 39(4): 521-535.

Allison, Paul D. 2009. Fixed effects regression models. Vol. 160. SAGE publications, 2009.

Baschieri, Angela, Kazuyo Machiyama, Sian Floyd, Albert Dube, Anna Molesworth, Menard Chihana, Judith R. Glynn, Amelia C. Crampin, Neil French, and John Cleland. 2017. Unintended childbearing and child growth in Northern Malawi, Maternal and Child Health Journal 21(3): 467-474. 
Bishai, David, Abdur Razzaque, Susan Christiansen, AHM Golam Mustafa, and Michelle Hindin. 2015. Selection bias in the link between child wantedness and child survival: theory and data from Matlab, Bangladesh, Demography 52(1): 61-82.

Boerma, J. Ties, and George T. Bicego. 1992. Preceding birth intervals and child survival: Searching for pathways of influence, Studies in Family Planning 23(4): 243-256.

Casterline, John B., and Colin Odden. 2016. Trends in inter-birth intervals in developing countries 1965-2014, Population and Development Review 42(2): 173-194.

Conde-Agudelo, Agustín, Anyeli Rosas-Bermudez, Fabio Castaño, and Maureen H. Norton. 2012. Effects of birth spacing on maternal, perinatal, infant, and child health: A systematic review of causal mechanisms, Studies in Family Planning 43(2): 93-114.

Conde-Agudelo, Agustin, José M. Belizán, Maureen H. Norton, and Anyeli Rosas-Bermúdez. 2005. Effect of the interpregnancy interval on perinatal outcomes in Latin America, Obstetrics \& Gynecology 106(2): 359-366.

DaVanzo, Julie, Lauren Hale, Abdur Razzaque, and Mizanur Rahman. 2008. The effects of pregnancy spacing on infant and child mortality in Matlab, Bangladesh: How they vary by the type of pregnancy outcome that began the interval, Population Studies 62(2): 131154.

Desai, Sonalde. 1992. Children at risk: The role of family structure in Latin America and West Africa. The Population and Development Review 689-717.

Dewey, Kathryn G., and Roberta J. Cohen. 2007. Does birth spacing affect maternal or child nutritional status? A systematic literature review, Maternal \& Child Nutrition 3(3): 151173.

Fenske, N., Burns, J., Hothorn, T., \& Rehfuess, E. A. (2013). Understanding child stunting in India: a comprehensive analysis of socio-economic, nutritional and environmental determinants using additive quantile regression. PloS one, 8(11).

Fotso, Jean Christophe, John Cleland, Blessing Mberu, Michael Mutua, and Patricia Elungata. 2013. Birth spacing and child mortality: An analysis of prospective data from the Nairobi urban health and demographic surveillance system, Journal of Biosocial Science 45(6): 779-798.

Gipson, Jessica D., Michael A. Koenig, and Michelle J. Hindin. 2008. The effects of unintended pregnancy on infant, child, and parental health: A review of the literature, Studies in Family Planning 39(1): 18-38.

Gutbrod, Tina, Dieter Wolke, Brigitte Soehne, Barbara Ohrt, and Klaus Riegel. 2000. Effects of gestation and birth weight on the growth and development of very low birthweight 
small for gestational age infants: A matched group comparison, Archives of Disease in Childhood-Fetal and Neonatal Edition 82(3): F208-F214.

Hoddinott, John, Harold Alderman, Jere R. Behrman, Lawrence Haddad, and Susan Horton. 2013. The economic rationale for investing in stunting reduction, Maternal \& Child Nutrition 9(Suppl.2): 69-82.

Horton, Susan. 1988. Birth order and child nutritional status: Evidence from the Philippines, Economic Development and Cultural Change 36(2): 341-354.

International Institute for Population Sciences (IIPS) and ICF. 2017. National Family Health Survey (NFHS-4), 2015-16: India. Mumbai: IIPS.

International Institute for Population Sciences (IIPS) and ORC Macro. 2000. National Family Health Survey (NFHS-2), 1998-99: India. Mumbai: IIPS.

International Institute for Population Sciences (IIPS). 1995. National Family Health Survey (MCH and Family Planning), India 1992-93, Bombay: IIPS.

International Institute for Population Sciences. 2017. National Family Health Survey-4: Fact Sheet (2015-16). http://rchiips.org/NFHS/pdf/NFHS4/India.pdf.

International Institute for Population Studies (IIPS) \& Macro International. 2007. National Family Health Survey (NFHS-3), 2005-06: India: Volume I. IIPS, Mumbai.

Jayachandran, S., \& Pande, R. (2017). Why are Indian children so short? The role of birth order and son preference. American Economic Review, 107(9), 2600-2629.

Marston, Cicely, and John Cleland. 2003. Do unintended pregnancies carried to term lead to adverse outcomes for mother and child? An assessment in five developing countries, Population Studies 57(1): 77-93.

McFadden, Daniel L. 1984. Econometric analysis of qualitative response models. Handbook of econometrics, 2, 1395-1457.

Molitoris, Joseph. 2018. Heterogeneous effects of birth spacing on neonatal mortality risks in Bangladesh, Studies in Family Planning 49(1): 3-21.

Montgomery, Mark R., and Cynthia B. Lloyd. 1997. Excess fertility, unintended births, and children's schooling. New York; Population Council, Policy Research Division Working Paper 100.

Öberg, Stefan. 2017. Too many is not enough: studying how children are affected by their number of siblings and resource dilution in families. 157-174.

Rahman, Md Mosfequr. 2015. Is unwanted birth associated with child malnutrition in Bangladesh? International perspectives on sexual and reproductive health 41(2): 80-88. 
Rana, Md Juel, and Srinivas Goli. 2018. Does planning of births affect childhood undernutrition? Evidence from demographic and health surveys of selected South Asian countries, Nutrition 47(March): 90-96.

Roy, Tarun K., R. K. Sinha, Michael Koenig, Sanjay K. Mohanty, and Sangram K. Patel. 2008. Consistency and predictive ability of fertility preference indicators: Longitudinal evidence from rural India, International Family Planning Perspectives 34(3): 138-145.

Rutstein, Shea O. 2005. Effects of preceding birth intervals on neonatal, infant and under-five years mortality and nutritional status in developing countries: Evidence from the demographic and health surveys, International Journal of Gynecology \& Obstetrics 89(S1): S7-S24.

Rutstein, Shea O., and Rebecca Winter. 2014. The effects of fertility behavior on child survival and child nutritional status: Evidence from the Demographic and Health Surveys 2006 to 2012, DHS Analytical Studies No. 37. Rockville, Maryland, USA: ICF International.

Schaible, Ulrich E., and H. E. Stefan. 2007. Malnutrition and infection: Complex mechanisms and global impacts, PLoS Medicine 4(5): e115.

Shahjada, A., Sharma, B. K., Sharma, S., Mahashabde, P., \& Bachhotiya, A. (2014). Effects of birth interval on nutritional status of under five children in periurban area of Madhya Pradesh, India. International Journal of Medical Science and Public Health, 3(6), 723727.

Shaka, Mohammed Feyisso, Yetayal Birhanu Woldie, Hirbaye Mokona Lola, Kalkidan Yohannes Olkamo, and Adane Tesfaye Anbasse. 2020. Determinants of undernutrition among children under-five years old in southern Ethiopia: does pregnancy intention matter? A community-based unmatched case-control study. BMC pediatrics 20(1): 1-10.

Singh, Abhishek, Ashish Kumar Upadhyay, Ashish Singh, and Kaushalendra Kumar. 2017. The association between unintended births and poor child development in India: Evidence from a longitudinal study, Studies in Family Planning 48(1): 55-71.

Singh, Abhishek, Ashish Singh, and Bidhubhusan Mahapatra. 2013. The consequences of unintended pregnancy for maternal and child health in rural India: Evidence from prospective data, Maternal and Child Health Journal 17(3): 493-500.

Singh, Abhishek, Satvika Chalasani, Michael A. Koenig, and Bidhubhusan Mahapatra. 2012. The consequences of unintended births for maternal and child health in India, Population Studies 66(3): 223-239. 
Singh, Susheela, Chander Shekhar, Rajib Acharya, Ann M. Moore, Melissa Stillman, Manas R. Pradhan, Jennifer J. Frost et al. 2018. The incidence of abortion and unintended pregnancy in India, 2015, The Lancet Global Health 6(1): e111-e120.

Spears, D., Coffey, D., \& Behrman, J. R. (2019). Birth Order, Fertility, and Child Height in India and Africa (No. 12289). Institute for the Study of Labor (IZA).

Speizer, Ilene S., Lisa M. Calhoun, Theresa Hoke, and Ranajit Sengupta. 2013. Measurement of unmet need for family planning: Longitudinal analysis of the impact of fertility desires on subsequent childbearing behaviors among urban women from Uttar Pradesh, India, Contraception 88(4): 553-560.

Srinivasan, Krishnamurthy. 2017. Population concerns in India: Shifting trends, policies, and programs. SAGE Publishing India.

Srivastava, Anurag, Syed E. Mahmood, Payal M. Srivastava, Ved P. Shrotriya, and Bhushan Kumar. 2012. Nutritional status of school-age children-a scenario of urban slums in India, Archives of Public Health 70(1): 1-8.

United Nations Children's Fund (UNICEF). 2017. Undernutrition contributes nearly half of all deaths in children under five and is widespread in Asia and Africa, https://data.unicef.org/topic/nutrition/malnutrition/. (Accessed 11/12/2017).

Victora, Cesar G., Linda Adair, Caroline Fall, Pedro C. Hallal, Reynaldo Martorell, Linda Richter, Harshpal Singh Sachdev, and Maternal and Child Undernutrition Study Group. 2008. Maternal and child undernutrition: Consequences for adult health and human capital, The lancet 371(9609): 340-357.

WHO Multicentre Growth Reference Study Group, and Mercedes de Onis. 2006. WHO child growth standards based on length/height, weight and age, Acta Paediatrica 95 (Supll. 450): 76-85.

Yaya, Sanni, Olanrewaju Oladimeji, Emmanuel Kolawole Odusina, and Ghose Bishwajit. 2020. Household structure, maternal characteristics and children's stunting in sub-Saharan Africa: evidence from 35 countries. International Health. 


\section{Tables}

Table 1: Descriptive statistics of the sample size for the key study variables and prevalence of child stunting in India, 2015-16

\begin{tabular}{lccc}
\hline \multirow{2}{*}{ Variables } & \multicolumn{2}{c}{ Sample } & Prevalence of stunting \\
\cline { 2 - 4 } & $\mathrm{n}$ & $\%$ & $\%[95 \% \mathrm{CI}]$ \\
\hline Preceding birth interval (months) & 28,249 & 13.9 & $35.8[35.2,36.4]$ \\
$>48$ & 23,241 & 11.4 & $41.9[41.3,42.6]$ \\
$37-48$ & 41,058 & 20.2 & $45.5[45.0,46.0]$ \\
$25-36$ & 33,845 & 16.6 & $48.8[48.3,49.4]$ \\
$<25$ & 75,481 & 37.1 & $34.4[34.0,34.7]$ \\
$\quad$ First birth order & & & \\
Succeeding birth & $1,48,066$ & 73.3 & $38.0[37.7,38.2]$ \\
$\quad$ No & 55,247 & 26.7 & $45.4[45.0,45.9]$ \\
Yes & & & \\
Sibsize (number of living children) & 50,267 & 24.7 & $32.0[31.6,32.4]$ \\
1 & 78,009 & 38.4 & $37.6[37.3,37.9]$ \\
2 & 40,823 & 20.1 & $45.5[45.0,46.0]$ \\
3 & 18,707 & 9.2 & $51.1[50.3,51.9]$ \\
4 & 15,507 & 7.6 & $55.4[54.6,56.3]$ \\
$5+$ & & & \\
Intendedness of births & $1,82,531$ & 89.8 & $39.5[39.3,39.7]$ \\
$\quad$ Wanted then (wanted) & 9,890 & 4.9 & $40.6[39.6,41.6]$ \\
$\quad$ Wanted later (mistimed) & 10,892 & 5.4 & $48.0[47.0,48.9]$ \\
Wanted no more (unwanted) & & & \\
Difference between desired and actual & & & \\
number of children & 148,015 & 73.2 & $37.7[37.5,38.0]$ \\
DFS $\geq$ AFS & 54,093 & 26.8 & $46.1[45.7,46.6]$ \\
DFS $<$ AFS & & &
\end{tabular}

Note: CI stands for confidence interval; DFS: Desired Family Size; AFS: Actual Family Size; The category of DFS $\geq$ AFS excludes 1,205 cases where mothers reported their desired family size in nonnumeric forms; The sample with less than 7 months and more than 10 years of preceding birth interval were dropped $(n=1,439)$. 
Table 2: Unadjusted and adjusted ORs of birth interval and intendedness of births on child stunting in India, 2015-16 $(n=201,874)$

\begin{tabular}{|c|c|c|c|}
\hline Variables & $\begin{array}{l}\text { Unadjusted OR }{ }^{1} \\
{[95 \% \mathrm{CI}]}\end{array}$ & $\begin{array}{l}\text { Adjusted OR }{ }^{2} \\
{[95 \% \mathrm{CI}]}\end{array}$ & $\begin{array}{l}\text { Adjusted } \mathrm{OR}^{3} \\
{[95 \% \mathrm{CI}]}\end{array}$ \\
\hline \multicolumn{4}{|c|}{ Preceding birth interval (months) } \\
\hline$>48$ & 1.00 & 1.00 & 1.00 \\
\hline $37-48$ & $1.30[1.25,1.35]$ & $1.27[1.22,1.31]$ & $1.13[1.08,1.17]$ \\
\hline $25-36$ & $1.50[1.45,1.55]$ & $1.45[1.40,1.49]$ & $1.27[1.22,1.31]$ \\
\hline$<25$ & $1.71[1.66,1.77]$ & $1.63[1.58,1.69]$ & $1.45[1.40,1.50]$ \\
\hline First birth order & $0.94[0.91,0.97]$ & $0.87[0.84,0.89]$ & $1.07[1.02,1.12]$ \\
\hline \multicolumn{4}{|l|}{ Succeeding birth } \\
\hline No & 1.00 & 1.00 & 1.00 \\
\hline Yes & $1.35[1.33,1.39]$ & $1.45[1.42,1.48]$ & $1.02[0.99,1.05]$ \\
\hline \multicolumn{4}{|c|}{ Intendedness of births } \\
\hline Wanted & 1.00 & 1.00 & 1.00 \\
\hline Mistimed & $1.05[1.00,1.09]$ & $0.96[0.91,1.00]$ & $1.00[0.96,1.05]$ \\
\hline Unwanted & $1.41[1.36,1.47]$ & $1.22[1.17,1.27]$ & $1.01[0.97,1.06]$ \\
\hline \multicolumn{4}{|c|}{$\begin{array}{l}\text { Note: OR: Odds Ratios; Mistimed and unwanted represent births which are reported as 'wanted later' and } \\
\text { 'wanted no more' at the time of survey; } 95 \% \text { confidence intervals are shown in the parentheses; }\end{array}$} \\
\hline \multirow{3}{*}{\multicolumn{4}{|c|}{$\begin{array}{l}\text { The sample size for this study excludes births whose preceding birth interval are less than seven months and } \\
\text { more than ten years } \\
{ }^{1} \text { Three separate binary logistic regression models were applied for preceding birth interval, succeeding birth, } \\
\text { and intendedness of births respectively } \\
2 \text {. }\end{array}$}} \\
\hline & & & \\
\hline \multicolumn{2}{|c|}{${ }^{2}$ Adjusted for birth interval and birth intendedness } & & \\
\hline \multicolumn{4}{|c|}{$\begin{array}{l}3 \text { Adjusted for age and sex of the child, age of mother at birth of the index child, number of living children, } \\
\text { mother's height, education, working status, place of residence, religion, caste, wealth status of the households } \\
\text { and regions }\end{array}$} \\
\hline
\end{tabular}


Table 3: Unadjusted and adjusted ORs of sibsize and difference between desired and actual number of children on child stunting in India, 2015-16 $(n=202,108)$

\begin{tabular}{|c|c|c|c|}
\hline Variables & $\begin{array}{c}\text { Unadjusted OR }^{1} \\
{[95 \% \mathrm{CI}]}\end{array}$ & $\begin{array}{c}\text { Adjusted OR }{ }^{2} \\
{[95 \% \mathrm{CI}]}\end{array}$ & $\begin{array}{c}\text { Adjusted } \mathrm{OR}^{3} \\
{[95 \% \mathrm{CI}]}\end{array}$ \\
\hline \multicolumn{4}{|l|}{ Sibsize } \\
\hline 1 (Ref.) & 1.00 & 1.00 & 1.00 \\
\hline 2 & $1.28[1.25,1.31]$ & $1.29[1.26,1.33]$ & $1.08[1.04,1.13]$ \\
\hline 3 & $1.77[1.73,1.82]$ & $1.89[1.83,1.95]$ & $1.22[1.16,1.29]$ \\
\hline 4 & $2.22[2.14,2.31]$ & $2.42[2.32,2.52]$ & $1.35[1.27,1.44]$ \\
\hline $5+$ & $2.65[2.54,2.76]$ & $2.95[2.81,3.09]$ & $1.52[1.42,1.63]$ \\
\hline \multicolumn{4}{|c|}{ Difference between desired and actual number of children } \\
\hline DFS $\geq$ AFS (Ref.) & 1.00 & 1.00 & 1.00 \\
\hline $\mathrm{DFS}<\mathrm{AFS}$ & $1.41[1.38,1.44]$ & $0.88[0.86,0.91]$ & $0.93[0.91,0.96]$ \\
\hline
\end{tabular}

Note: OR: Odds Ratios; $95 \%$ confidence intervals are shown in the parentheses;

${ }^{1}$ Two separate binary logistic regression models were applied for sibsize and difference between desired and actual family size respectively

${ }^{2}$ Adjusted for desired vs actual family size and actual family size;

${ }^{3}$ Adjusted for age and sex of the child, age of mother at birth of the index child, preceding birth interval, succeeding birth, mother's height, education, working status, place of residence, religion, caste, wealth status of the households and regions 
Table 4: Unadjusted and adjusted ORs of stunting by sibsize, according to the difference between actual and desired family size in India, 2015-16 $(n=202,108)$

\begin{tabular}{lll}
\hline \multicolumn{1}{c}{ Sibsize } & DFS $\geq$ AFS & DFS $<$ AFS \\
\hline Unadjusted ORs & & \\
1 (Ref.) & 1.00 & 1.00 \\
2 & $1.28[1.25,1.32]$ & $1.39[1.22,1.58]$ \\
3 & $1.98[1.91,2.05]$ & $1.82[1.61,2.05]$ \\
4 & $2.40[2.25,2.55]$ & $2.43[2.15,2.75]$ \\
$5+$ & $2.47[2.21,2.75]$ & $3.01[2.66,3.41]$ \\
Adjusted ORs & & \\
1 (Ref.) & 1.00 & 1.00 \\
2 & $1.09[1.04,1.15]$ & $1.24[1.07,1.42]$ \\
3 & $1.21[1.13,1.31]$ & $1.45[1.26,1.68]$ \\
4 & $1.36[1.24,1.49]$ & $1.58[1.36,1.83]$ \\
$5+$ & $1.46[1.27,1.67]$ & $1.77[1.52,2.06]$ \\
\hline
\end{tabular}

Note: Ref. denotes reference category; OR: Odds Ratios; $95 \%$ confidence intervals are shown in the parentheses; Adjusted for age and sex of the child, age of mother at birth of the index child, preceding birth interval, succeeding birth, mother's height, education, working status, place of residence, religion, caste, wealth status of the households and regions 
Table 5: Effects of preceding birth interval on childhood stunting in India $(n=30,997)$

\begin{tabular}{lll}
\hline \multirow{2}{*}{ Preceding birth interval (months) } & \multicolumn{2}{c}{ Adjusted OR [95\% CI] } \\
\cline { 2 - 3 } & $\begin{array}{c}\text { Without mother-level } \\
\text { fixed effects }\end{array}$ & $\begin{array}{c}\text { With mother-level fixed } \\
\text { Effects }^{2}\end{array}$ \\
\hline$>48$ (Ref.) & 1.00 & 1.00 \\
$37-48$ & $1.14[0.99,1.32]$ & $1.36[1.16,1.59]$ \\
$25-36$ & $1.41[1.24,1.62]$ & $1.34[1.16,1.55]$ \\
$<25$ & $2.25[1.96,2.57]$ & $1.79[1.55,2.07]$ \\
First birth order & $1.32[1.15,1.51]$ & $1.29[1.12,1.48]$ \\
\hline
\end{tabular}

Note: The fixed effects sample are the children whose mothers have at least two second or higher order births delivered in the last five years of the survey and also have at least one positive outcome (at least one child is stunted and not stunted); Out of total 14,815 mothers, 13,462 mothers have two children, 1,335 mothers have three children, 16 mothers have four children.

OR: Odds Ratios; The reference category is $>48$ months of birth interval; Adjusted for number of living children, age and sex of the child, age of mother at birth of the index child; Confidence interval at $95 \%$ confidence level has been reported in the parentheses

${ }^{1}$ Results from binary logistic model

${ }^{2}$ Results from conditional binary logistic model or mother-level fixed effect model 


\section{Appendix Figure}

Figure A1: Preceding birth interval (in months) in India from 1992-93 to 2015-16

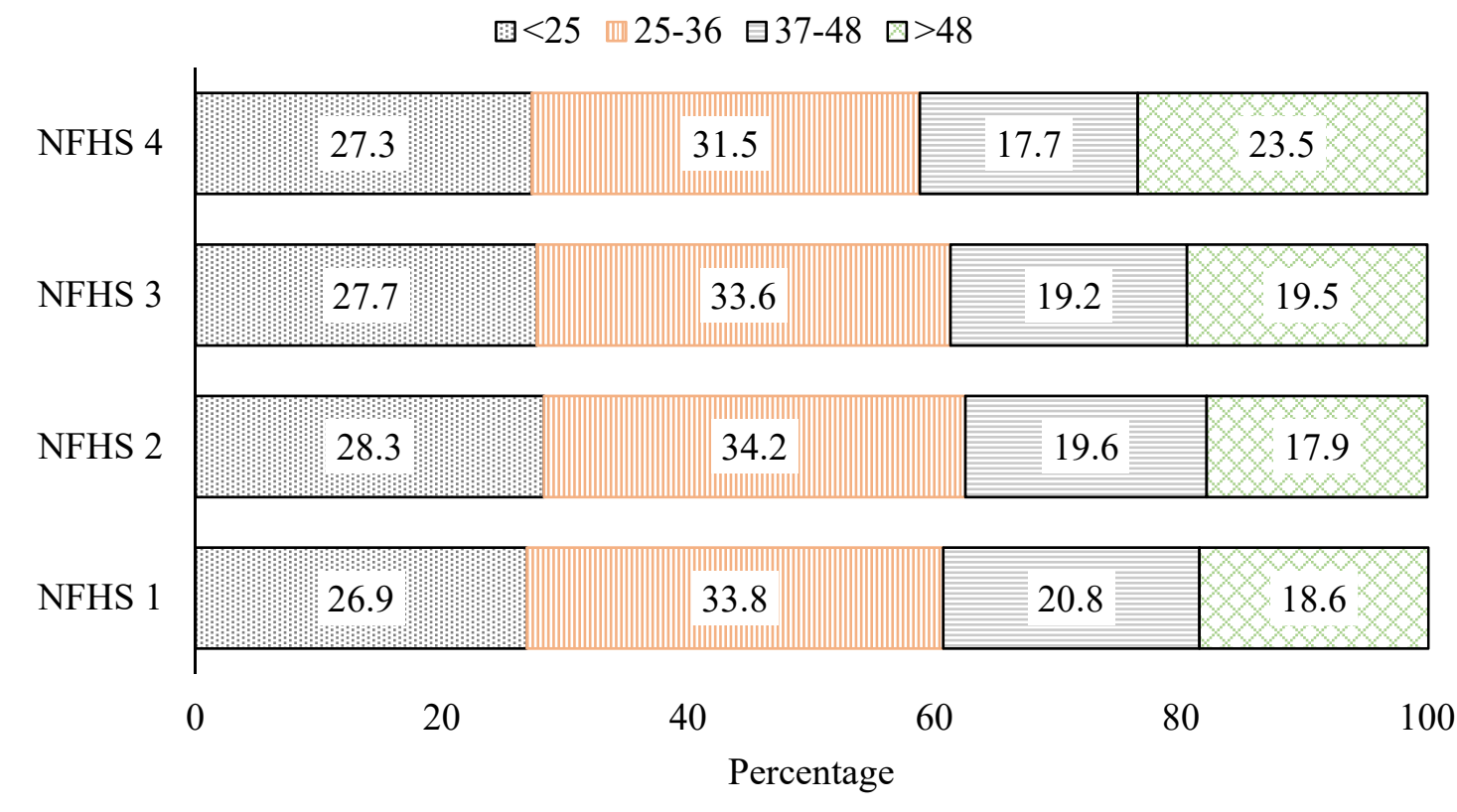

Source: National Family Health Survey Reports (NFHS 1, 2, 3 and 4) 
Table A1: Percentage of mistimed and unwanted births by preceding birth interval, and AFS-DFS by sibsize in India, 2015-16

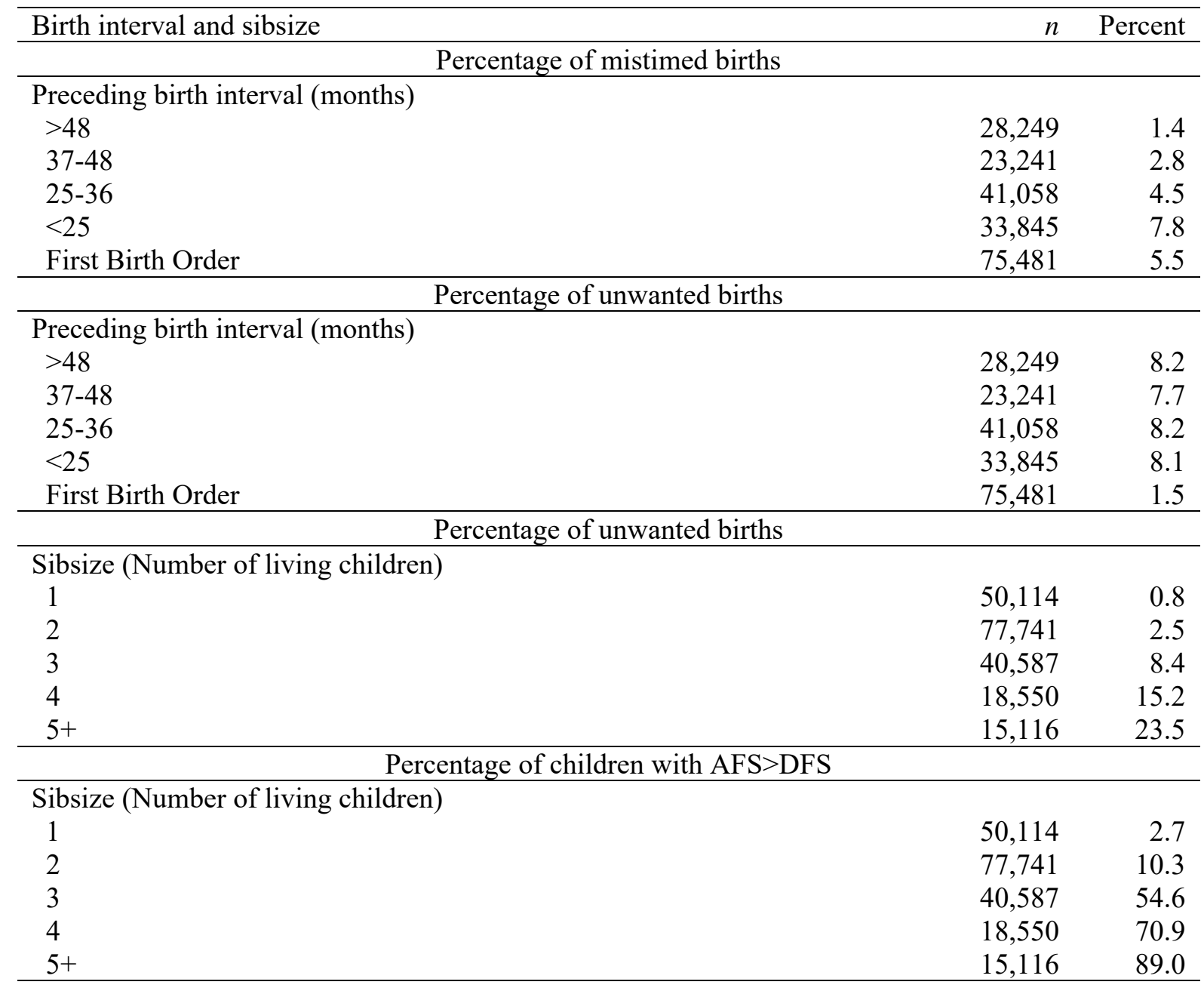

Note: CI stands for $95 \%$ confidence interval 
Table A2: Univariate descriptive statistics of the sample and percentage of child stunting in India, 2015-16

\begin{tabular}{|c|c|c|c|}
\hline \multirow{2}{*}{ Variables } & \multicolumn{2}{|c|}{$\begin{array}{l}\text { Univariate descriptive } \\
\text { statistics }\end{array}$} & \multirow{2}{*}{$\begin{array}{c}\begin{array}{c}\text { Prevalence of } \\
\text { stunting }\end{array} \\
\%[95 \% \mathrm{CI}]\end{array}$} \\
\hline & $n$ & $\%$ & \\
\hline \multicolumn{4}{|l|}{ Childhood stunting } \\
\hline Normal & 122088 & 60.1 & - \\
\hline Stunted & 81225 & 39.9 & - \\
\hline \multicolumn{4}{|c|}{ Age of the child (months) } \\
\hline $6-11$ & 22,618 & 11.1 & $22.8[22.3,23.4]$ \\
\hline $12-23$ & 44,678 & 22.0 & $42.6[42.1,43.0]$ \\
\hline $24-35$ & 44,520 & 21.9 & $42.7[42.2,43.2]$ \\
\hline $36-47$ & 46,725 & 23.0 & $43.2[42.7,43.6]$ \\
\hline $48-59$ & 44,772 & 22.0 & $39.9[39.4,40.3]$ \\
\hline \multicolumn{4}{|l|}{ Sex of the child } \\
\hline Male & $1,05,311$ & 51.8 & $40.3[40.0,40.6]$ \\
\hline Female & 98,002 & 48.2 & $39.5[39.2,39.9]$ \\
\hline \multicolumn{4}{|c|}{$\begin{array}{l}\text { Age of mother at birth of the index child } \\
\text { (years) }\end{array}$} \\
\hline$<20$ & 24957 & 13.7 & $42.2[41.6,42.8]$ \\
\hline $20-24$ & 88843 & 45.6 & $39.6[39.3,39.9]$ \\
\hline $25-29$ & 57498 & 27.3 & $38.4[38.0,38.8]$ \\
\hline $30+$ & 32015 & 13.4 & $42.0[41.4,42.6]$ \\
\hline \multicolumn{4}{|l|}{ Mother's height (cm) } \\
\hline$<145$ & 23,131 & 11.4 & $59.9[59.2,60.5]$ \\
\hline 145 and above & $1,79,918$ & 88.5 & $37.3[37.1,37.5]$ \\
\hline Not measured & 264 & 0.1 & $41.2[35.7,47.0]$ \\
\hline \multicolumn{4}{|l|}{ Mother's education } \\
\hline No education & 63,107 & 31.0 & $52.7[52.3,53.1]$ \\
\hline Primary & 29,871 & 14.7 & $45.3[44.7,45.9]$ \\
\hline Secondary & 91,734 & 45.1 & $34.0[33.7,34.3]$ \\
\hline Higher & 18,601 & 9.1 & $21.4[20.9,22.0]$ \\
\hline \multicolumn{4}{|l|}{ Working status $^{1}$} \\
\hline Not working & 29,053 & 14.3 & $38.7[38.1,39.3]$ \\
\hline Working & 6,397 & 3.1 & $41.6[40.3,42.9]$ \\
\hline Not reported/asked & $1,67,863$ & 82.6 & $40.1[39.9,40.3]$ \\
\hline \multicolumn{4}{|l|}{ Place of residence } \\
\hline Urban & 48,482 & 23.8 & $31.9[31.5,32.3]$ \\
\hline Rural & $1,54,831$ & 76.2 & $43.1[42.8,43.3]$ \\
\hline \multicolumn{4}{|l|}{ Religion } \\
\hline Hindu & $1,47,257$ & 72.4 & $40.0[39.8,40.3]$ \\
\hline Muslim & 31,834 & 15.7 & $41.6[41.1,42.2]$ \\
\hline Others & 24,222 & 11.9 & $32.9[31.9,33.8]$ \\
\hline \multicolumn{4}{|l|}{ Caste } \\
\hline Others & 35,572 & 17.5 & $31.7[31.3,32.2]$ \\
\hline $\mathrm{SC}$ & 38,574 & 19.0 & $44.5[44.0,45.0]$ \\
\hline ST & 40,017 & 19.7 & $45.8[45.1,46.5]$ \\
\hline $\mathrm{OBC}$ & 80,226 & 39.5 & $40.4[40.0,40.7]$ \\
\hline Not reported & 8,924 & 4.4 & $35.9[34.9,37.0]$ \\
\hline
\end{tabular}


Table A2: Univariate descriptive statistics of the sample and percentage of child stunting in India, 2015-16 (Contd.)

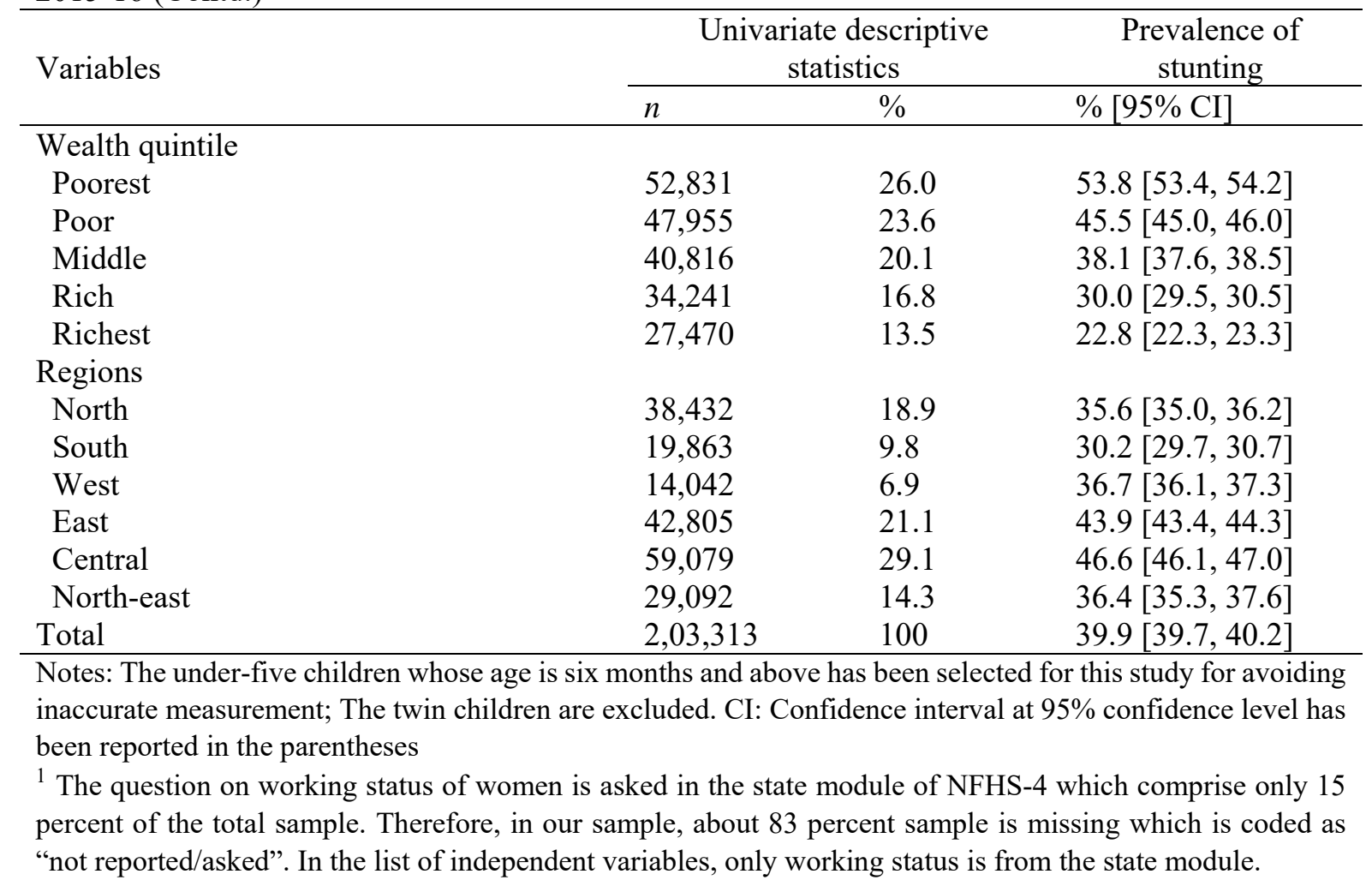

